applied. But in general the states are not orthogonal to each other, so even though we know the list of possible states and their a priori probabilities, there is no reliable way of identifying the signals with certainty. What is the minimum number of resources - the physical bits - for the coding in this case? Here the physical bit is any quantum object for which the state is described by a vector in two-dimensional Hilbert space. Quantum physical bits are also called 'qubits', so we are trying to find the minimum number of qubits per quantum symbol.

Schumacher's new work ${ }^{5}$ has proved that there is a lower bound of number of qubits per symbol and this bound can be less than Shannon's entropy. The quantity that determines the bound is known as von Neumann entropy and depends both on the $a$ priori probabilities of the symbols and on the vectors representing the symbol states. The von Neumann entropy is smaller than the Shannon entropy except when the symbol states are mutually orthogonal, in which case the two entropies are equal.

One way to think about the quantum generalization of the Shannon theorem is to consider the coding of longer and longer blocks of symbols. If each symbolstate is described by a vector in the $\mathrm{N}$-dimensional Hilbert space then a block of $k$ state-symbols can be described as one vector, but this time in $N^{k}$-dimensional Hilbert space. Because there are $n$ symbols, then each block of $k$ symbols can be in one of the $n^{k}$ states, each state being a vector in the $N^{k}$-dimensional Hilbert space. But because of the different probabilities associated with different symbols, some blocks of symbols will have negligible probabilities of occurrence. It turns out that the vectors representing the typical blocks with the non-negligible probabilities lie with pretty good accuracy in the $2^{D k}$-dimensional subspace of the $N^{k}$ dimensional Hilbert space where $D$ is the von Neumann entropy of the source. That is why, for long blocks, we need on average about $D$ qubits per symbol.

This generalization of the Shannon theorem comes just in time. The technology is ripening to the stage where it can support quantum data processing. Experimental progress in quantum optics enabled us to construct the first quantum cryptosystems. If quantum computation is to be the next experimental target, quantum coding will be essential.

Artur Ekert is at the Clarendon Laboratory, University of Oxford, Parks Road, Oxford OX1 3PU, UK.

1. Stewart, I. Nature 353, 384 (1991)

2. Ekert, A. Nature 358, 14 (1992).

3. Sudbery, T. Nature 362, 586 (1993).

Bennett, C. H. Nature 362, 694 (1993).

. Schumacher, B. Phys. Rev. A (in the press).

6. Welsh, D. Codes and Cryptography (Clarendon, Oxford 1988).

\title{
From models to reality
}

\section{MichaelF. Sheridan}

IN a volcano's eruptive repertoire, some of the most violent phenomena are the giant plinian eruption columns that pierce the tropopause and their associated lateral pulses of hot mixtures of particles and gas. They not only devastate inhabited areas near the volcanoes but may also affect climate worldwide. Understanding the physics of such events and quantifying the movement of the ash clouds associated with giant volcanic eruptions has been the goal of volcanological research during the past quarter of a century.
The most ambitious and sophisticated numerical models of pyroclastic flow eruptions so far are being developed by the Gruppo Nazionale per la Volcanologia in Pisa. On page 551 of this issue, F. Dobran and colleagues demonstrate the models' abilities: shown here is a snapshot of detailed particle concentrations in a pyroclastic flow moving down the flanks of Vesuvius. This model realistically simulates the generation of an elutriation plume (also known as a phoenix cloud), in which the finer, lighter particles are borne

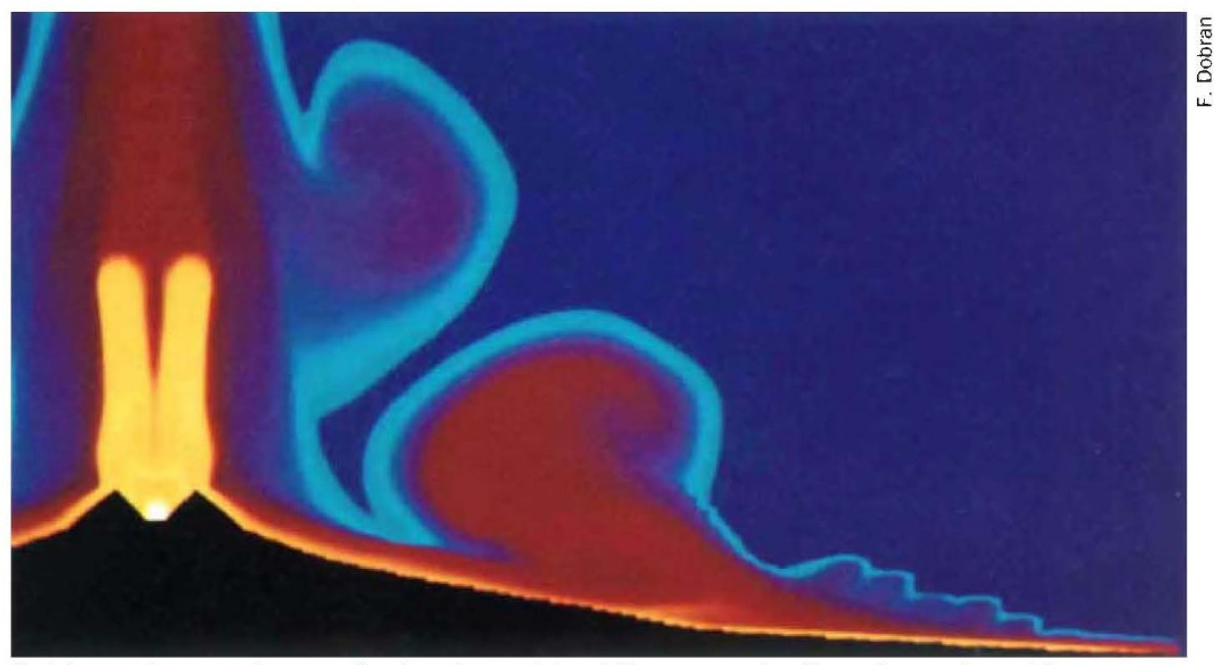

Raising a dust - plumes of volcanic particies billow upwards, five minutes into this computer simulation of Vesuvius in eruption.

One fruitful line of investigation is to use large computers to make dynamic models of multi-phase systems with the objective of realistically representing natural systems. An early attempt to model large volcanic explosions $(\mathrm{K} . \mathrm{H}$. Wohletz et al. J. geophys. Res. 89, 8269$8285 ; 1984)$ used a two-dimensional axisymmetric numerical simulation based on a shock tube analogy. The most important result was the quantification of parameters related to the initial blast wave produced during the first 60 seconds of giant volcanic explosions. Later models used time-dependent, two-phase compressible Navier-Stokes equations to simulate the first few minutes of continuous volcanic jets (G. A. Valentine and K. H. Wohletz J. geophys. Res. 94, $1867-$ 1887; 1989). These models mimicked the development of plinian eruption columns and clarified conditions that relate to column collapse and determine how material in pyroclastic flows will be distributed. By introducing large obstacles in the finite-element grid, Valentine and co-workers determined the effect of caldera margins on general flow properties (G. A. Valentine et al. Geol. Soc. Am. Bull. 104, 154-165; 1992). upwards by the air currents where the volcano slope flattens.

But how do we use such elegant models to reduce the loss of life near dangerous volcanoes? If Vesuvius were to produce an eruption with pyroclastic flows such as the one pictured here, between 250,000 and $2,500,000$ people would be in jeopardy. Evacuation of such numbers from the Naples area, even if they had ample warning, seems improbable. Ideally, future research on this question would bring in teams of policy makers, city planners, engineers, public safety officers and medical experts in addition to geoscientists. One goal of this effort could be to develop low-cost, efficient shelters that would be aerodynamically designed to withstand the heat and force of simulated flows. Hazard planning near volcanoes could then include an adequate number of safe areas, man-made or natural, close enough to the inhabited areas for people to be able to walk to shelter just for the short duration of the most dangerous periods.

Michael F. Sheridan is in the Department of Geology, State University of New York at Buffalo, New York 14260, USA. 